\title{
Effects of Counterions on Molecular Transport Across Liposome Bilayer: Probed by Second Harmonic Generation
}

\author{
Xiaoming Shang, Yan Liu, Elsa Yan, and Kenneth B. Eisenthal* \\ Department of Chemistry, Columbia University, New York, New York 10027
}

Received: June 1, 2001; In Final Form: October 1, 2001

\begin{abstract}
The transport rate of an organic cation, malachite green $(\mathrm{MG})$, across a unilamellar bilayer $(\sim 105 \mathrm{~nm})$ of the dioleoylphosphatidylglycerol (DOPG) liposome has been studied by the SHG technique. This is the first time to our knowledge that the effects of anions on molecular cation transport have been observed. Our studies show four results. First, in the presence of sodium chloride $(\mathrm{NaCl})$ or sodium bromide $(\mathrm{NaBr})$, the time constant for transport of MG across the DOPG bilayer increases with the increase in the concentration of the counterion (i.e., $\mathrm{Cl}^{-}$or $\mathrm{Br}^{-}$). Second, with the organic electrolytes, sodium citrate (NaCitrate) and sodium ethanesulfonate $\left(\mathrm{NaEtSO}_{3}\right)$, the transport rate is independent of the concentration of the counterion (i.e., Citrate ${ }^{-}$ and $\mathrm{EtSO}_{3}{ }^{-}$). Third, at the same counterion concentration, the transport rate depends on the species of the counterion used. The rate of MG transport is the slowest with $\mathrm{Cl}^{-}$, faster with $\mathrm{Br}^{-}$, and the fastest with the two organic counterions, $\mathrm{Citrate}^{-}$and $\mathrm{EtSO}_{3}{ }^{-}$. Last, at the low counterion concentration of $1 \mathrm{mM}$, the transport rates of MG were found to approach the same value for the four anions. A brief discussion of a possible mechanism is presented.
\end{abstract}

\section{Introduction}

Liposomes have been widely used to investigate membrane functions because of the similarity between a liposome bilayer and biological membranes. Studies of liposomes in various fields of science and technology, ${ }^{1-3}$ extend from basic studies of the shapes and size distribution of cells, reconstitution of protein membranes, to applications such as drug delivery systems, photoconversion of solar energy, chemical catalysis, and cosmetics. In all these applications, molecular transport kinetics across the liposome bilayer plays an important and basic role, which has been studied extensively for several decades. ${ }^{4-12}$ This includes the mechanism of transport, ${ }^{10,13,14}$ the effects of bilayer chemical composition, phase structure, ${ }^{15,16}$ chemical and electrical gradients across a phospholipid bilayer, ${ }^{17,18}$ as well as a variety of practical problems including time-controlled drug release using liposomes as delivery vehicles. ${ }^{19}$ However, studies of the effects of the electrolyte on molecular transport across the liposome bilayer have not been reported to our knowledge.

The effect of the counterion (anion) on the molecular transport rate of an organic cation across the liposome membrane is reported in the present work. We used an organic cation, malachite green $(\mathrm{MG})$, which has a charge of +1 , as the transport probe and dioleoylphosphatidylglycerol (DOPG) $(\sim 105 \mathrm{~nm})$ as a negatively charged liposome. The molecular structures of MG and the DOPG lipid are shown in Scheme 1. DOPG consists of two unsaturated 18-carbon chains with one double bond located between the $9^{\text {th }}$ and the $10^{\text {th }}$ carbons in each carbon chain.

We used second harmonic generation (SHG) to measure the transport rates of $\mathrm{MG}$ across the liposome bilayer as a function of electrolyte concentration in four solutions with the same cation $\left(\mathrm{Na}^{+}\right)$and different anions $\left(\mathrm{Cl}^{-}, \mathrm{Br}^{-}, \mathrm{Citrate}^{-}\right.$, and $\mathrm{EtSO}_{3}{ }^{-}$). Our results show that the different counterions (i.e., $\mathrm{Cl}^{-}, \mathrm{Br}^{-}, \mathrm{Citrate}^{-}$, and $\mathrm{EtSO}_{3}{ }^{-}$) affect the $\mathrm{MG}$ transport rate in different ways. For example, the transport rate is the slowest with $\mathrm{Cl}^{-}$, faster with $\mathrm{Br}^{-}$, and the fastest with $\mathrm{Citrate}^{-}$and $\mathrm{EtSO}_{3}{ }^{-}$. In addition, we observed a concentration dependence of the transport rate with the two inorganic anions, $\mathrm{Cl}^{-}$and

* To whom correspondence should be addressed.

\section{SCHEME 1}

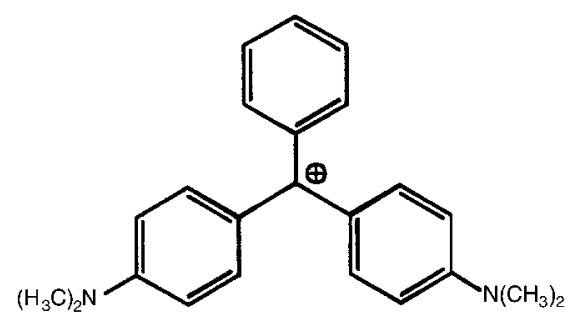

Malachite green cation (MG)

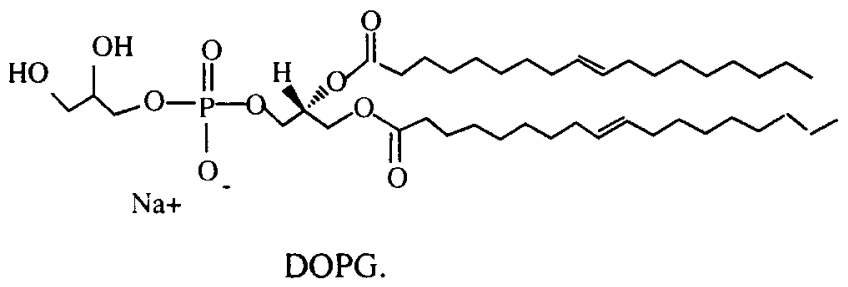

$\mathrm{Br}^{-}$. More specifically, the transport rate of MG across the DOPG liposome bilayer becomes slower when increasing the concentration of the counterion $\left(\mathrm{Cl}^{-}\right.$or $\left.\mathrm{Br}^{-}\right)$. However, for the two organic anions (Citrate ${ }^{-}$and $\mathrm{NaEtSO}_{3}{ }^{-}$), the transport is independent of their concentration, and the time constants of MG transport for these two organic electrolytes are the same within our experimental precision.

\section{SHG as a Probe of Ion Transport Kinetics across a Liposome Bilayer}

Second harmonic generation ( $\mathrm{SHG}$ ) is a second-order nonlinear optical effect, in which the incident light field at frequency $\omega$ interacts with the medium to generate a light wave at $2 \omega$. As a second-order process, it is forbidden in centrosymmetric media in the electric dipole approximation but is allowed at surfaces or interfaces, where the centrosymmetry is broken. This leads to a unique property of SHG, namely, its surface specificity. Some excellent reviews summarize recent progress in this field. ${ }^{20-25}$ 
In past work, we have shown that SHG can be used to study the surfaces of centrosymmetric microparticles ${ }^{26}$ as well as planar surfaces. The idea behind this method is that although the microscopic particle is centrosymmetric on a macroscopic scale, its surface is not locally centrosymmetric. Therefore, SHG can be generated from this kind of material provided the size of the microparticle is comparable to the coherence length of the SHG, i.e., roughly the wavelength of the SH light. ${ }^{22-33}$

One of the promising applications of this method is to investigate transport kinetics of molecules across liposome bilayers. ${ }^{28,31,33}$ Liposomes are spherical self-assembled bilayer structures that separate two aqueous phases. The phospholipids arrange in such a way that the nonpolar (hydrophobic) carbon chains escape the aqueous phase by forming a bilayer with the chains of the two layers facing each other, whereas the polar heads (for DOPG, the phosphatidylglycerol group) reside at the boundary with the surrounding aqueous solution. The thickness of the bilayer is 4-5 nanometers. Upon adding probe molecules to a liposome solution, some of the molecules are rapidly adsorbed onto the outer surface of the liposome and give a SHG signal. Let us define this time as time zero $(t=0)$. The adsorbed molecules can transport across the liposome bilayer driven by chemical and electrical forces and be adsorbed onto the inner surface of the bilayer. The molecules adsorbed on the inner surface are oriented opposite to those adsorbed on the outer surface because of symmetry. As a result, the second-order fields induced by the molecules adsorbed on the inner and outer liposome have opposite phases and cancel each other because the surfaces are separated by distances much less than the coherence length of the SHG process. This leads to a drop in the SHG signal as the MG cations cross to the inner surface. The number of molecules adsorbed on the outer surface is larger than that on the inner surface because the adsorbing area of the outer surface is larger than that of the inner surface. As a consequence there is not complete cancellation of the SHG signal. The SHG field $E_{2 \omega}$ is proportional to the second-order susceptibility $\chi^{(2)}$, which contains information about the population of adsorbates. In this study, $\chi^{(2)}$ is proportional to the adsorbate population difference between the outer and inner surfaces of the bilayer at time $t$. Therefore, $E_{2 \omega}$ can be written as

$$
E_{2 \omega}(t) \propto\left[N_{\mathrm{o}}(t)-N_{\mathrm{i}}(t)\right]
$$

where $N_{\mathrm{o}}(t)$ and $N_{\mathrm{i}}(t)$ are the adsorbate population densities at the outer and inner surfaces, respectively, at the time $t$.

The experimental techniques that have been used to study molecular transport kinetics across a bilayer include NMR ${ }^{15,34}$ EPR spin labels, ${ }^{17,35}$ and fluorescence. ${ }^{36}$ These techniques are confronted with several problems. First, they cannot always distinguish the molecules adsorbed at the surface of a liposome bilayer from those in the bulk solution, or the surface at which they are located, outer versus inner. Second, they require precise calibration of the signal changes with the microenvironment (NMR line width broadening, EPR spin labeling), separation of fluorescence and absorption surface signals from the large bulk background signals. In addition, some of these techniques require quenchers and spectral-shifting reagents. The addition of quenchers and spectral-shifting reagents can perturb the liposome structure and change the surface microenvironment. In contrast, $\mathrm{SHG}$ is a surface-specific technique, which is only sensitive to molecules at the surface. Thus, there is no need to introduce the extraneous quenchers, spectral-shifting reagents, and to involve complex chemical handling. However SHG has its limits as well. Most importantly, it requires an interfacial species that can generate a detectable SH signal.

One reason why the organic cation MG was chosen was that previous studies showed that MG can permeate across a DOPG liposome bilayer ${ }^{28,31,33}$ and thus can act as a probe to investigate ion transport kinetics. Another reason is that it has an absorption band peaked at $424 \mathrm{~nm}$, which is in resonance with twice the frequency of the laser light, and therefore gives a resonant enhancement of the SHG signal.

\section{Experimental Section}

Four kinds of electrolyte solutions were involved in this study, namely, sodium chloride $(\mathrm{NaCl})$, sodium bromide $(\mathrm{NaBr})$, sodium citrate ( $\mathrm{NaCitrate}$ ), and sodium ethanesulfonate ( $\mathrm{Na}$ $\mathrm{EtSO}_{3}$ ). The solutions of $\mathrm{NaCl}, \mathrm{NaBr}$, and $\mathrm{NaEtSO}_{3}$ were obtained by dissolving the corresponding salts in double distilled water. The $\mathrm{pH}$ value of these solutions was adjusted to be 4.0. The NaCitrate solutions were prepared by mixing citric acid with $\mathrm{NaOH}$ solution, and their $\mathrm{pH}$ was adjusted to 4.0 . The concentrations of electrolytes used are 1, 10, 32, 66, and 100 $\mathrm{mM}$.

DOPG phospholipid (Avanti Polar Lipids, Inc) was used to make a negatively charged liposome. Four steps were followed to prepare the liposome sample. First, the weighed DOPG lipid powder was dissolved in $5 \mathrm{~mL}$ chloroform. The solvent (chloroform) was then evaporated under vacuum in a rotavapor setup for more than $4 \mathrm{~h}$ to obtain a thin film. Next, the proper amount of salt solution was added to hydrate the thin film with vigorous vortexing. The concentration of the lipid was maintained at $2.5 \mathrm{mM}$. Mutilamellar vesicles (MLVs) are then formed. To more easily extrude the MLVs, a step called freezethawing is needed. Last, 10 cycles of extrusions were done with doubly stacked $0.2 \mu \mathrm{m}$ polycarbonate film at room temperature under a pressure of $110 \mathrm{psi}$. It has been demonstrated that more than $90 \%$ of liposomes are unilamellar after the extrusion procedure ${ }^{37}$ Then, the liposome solution was diluted 100 times in the salt solution for the SHG measurement. The liposome stock solution was stored at $4{ }^{\circ} \mathrm{C}$ whenever not used. The sizes of liposomes were obtained from UV-vis turbidity or dynamic light scattering measurements. ${ }^{38,39}$ All the liposome samples used in this study were very close in size, ranging from 105 to 110 $\mathrm{nm}$ in diameter.

The probe molecule, malachite green chloride was purchased from Aldrich Chemical Co. and was used as received after the purity was checked by the HPLC method. The malachite green chloride solutions were made consisting of four kinds of salt solutions. The concentration of malachite green chloride in all experiments was $20 \mu \mathrm{M}$. It should be noted that in aqueous solution, there exists an acid-base equilibrium between malachite green cation $(\mathrm{MG})$ and malachite green carbinol $(\mathrm{MGOH})$ (i.e., $\mathrm{MG}^{+}+\mathrm{H}_{2} \mathrm{O} \leftrightarrow \mathrm{MGOH}+\mathrm{H}^{+}$). A p $K_{\mathrm{a}}$ of $7.0 \pm 0.1$ for this equilibrium was derived from the absorption spectra of the bulk solutions at several $\mathrm{pH}$ values. In the bilayer transport experiments, we maintained the $\mathrm{pH}$ for all samples to be 4.0. This means, malachite green cation (denoted by MG) is the predominant form.

For the measurement of the transport kinetics, the liposome solution was mixed with an equal volume of MG solution, i.e., after mixing, the concentrations of both lipid and $\mathrm{MG}$ are diluted by a factor of 2 and are $12.5 \mu \mathrm{M}$ and $10 \mu \mathrm{M}$, respectively.

The experimental setup has been described in detail previously. ${ }^{31}$ The $90^{\circ}$ detection geometry is used. An Argon ion laser (Spectra-physics, Model 2080) pumped Ti:sappire oscillator (Spectra-physics, Tsunami) generates 100 fs pulses with an 


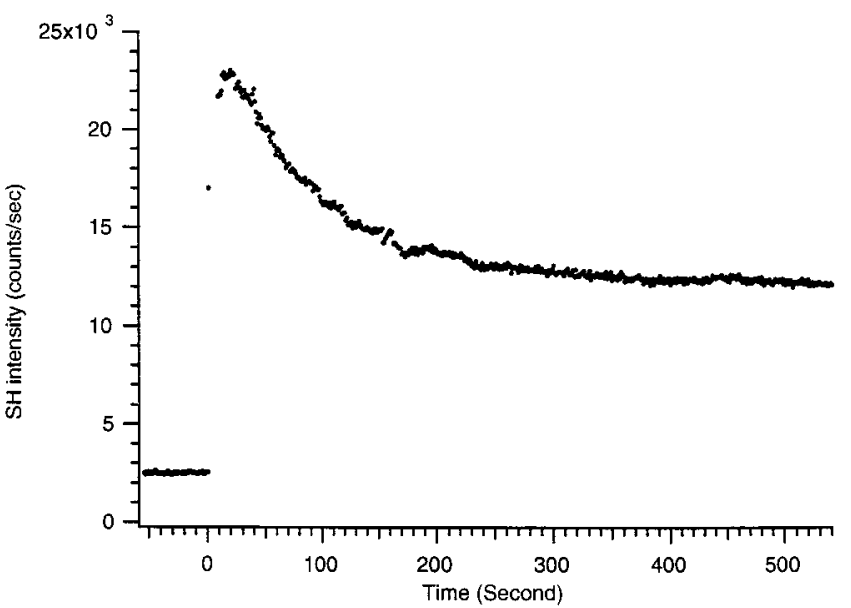

Figure 1. Temporal profile of SHG signal before and after injection of liposome into MG solution. The data shown is for an aqueous 10 $\mathrm{mM}$ solution of $\mathrm{NaCl}$ at $\mathrm{pH} 4.0$.

energy of $13 \mathrm{~nJ}$ per pulse. It was operated at $840 \mathrm{~nm}$ at a repetition rate of $82 \mathrm{MHz}$. The femtosecond laser pulse train passes through a half wave plate, a polarizer, and a color filter used to block all the stray light at twice the frequency of the laser light. It was then focused into the sample by a lens with a focal length of $5 \mathrm{~cm}$. The incident light was chosen to be $\mathrm{P}$ polarized. The generated SHG signal was collected at $90^{\circ}$ relative to incident light direction and sent into a monochromator. Before the monochromator, an analyzer was used to allow for selectively detecting the signal with the $\mathrm{P}$ polarization. The data acquisition system includes a PC connected with a PMT and a single photon counter. The addition of the liposome solution to the MG solution was completed within several seconds.

\section{Results and Discussions}

A. Kinetics of MG Crossing the Bilayer. The time evolution of the SHG signal on mixing the MG and DOPG liposome solutions was measured in the four electrolyte solutions (i.e., $\mathrm{NaCl}, \mathrm{NaBr}, \mathrm{NaCitrate}$, and $\mathrm{Na} \mathrm{EtSO}_{3}$ ) at several concentrations. The experimental curves for all the samples show similar features to that shown in Figure 1. Before adding liposome solution, the signal is nearly constant and is attributed to the two-photon fluorescence tail of MG and not hyper-Rayleigh scattering of water, as evidenced by the experimental finding that this signal is proportional to the concentration of the $\mathrm{MG}$ solution. The MG solution was used to calibrate the experimental data and to check the laser pump power from day to day. Immediately after the injection of the liposome solution into the MG solution, the signal shows a sharp rise followed by an exponential decay, whose decay time depends on the electrolyte used and its concentration. The sharp rise, limited by the injection process, is due to very fast adsorption of $\mathrm{MG}$ from the bulk MG solution onto the outer surface of the liposome bilayer. We define this time as time zero. Subsequently, MG will migrate across the DOPG bilayer and be adsorbed onto the inner surface of the membrane with an orientation opposite to that on the outer surface because of symmetry. As discussed in the Introduction, a decay of the SHG signal with time then occurs.

The analysis of the experimental data involves the background correction and calculation of the $\mathrm{SH}$ field, $E_{2 \omega}$. It can be described by the following equation

$$
E_{2 \omega}(t)=\sqrt{I_{\mathrm{MG}+\text { limpsome }}^{2 \omega}(t)-I_{\text {background }}}
$$

where $I_{\mathrm{MG}+\text { Limpsome }}^{2 \omega}(t)$ is the total signal detected at twice the frequency of the fundamental incident laser, $2 \omega$, at time $t$ after mixing of the MG solution and the liposome solution. $I_{\text {background }}$ represents the contributions from the factors other than the $\mathrm{SH}$ field generated by the MG adsorbed on the DOPG liposome bilayer. It is due mainly to the two-photon fluorescence tail of MG in the bulk solution. In the analysis, $I_{\text {background }}$ is the initial signal level before adding the liposome solution, which is divided by two because the concentration of MG is diluted by a factor of 2 after the injection of an equal volume of the liposome solution.

B. Dependence of SHG Decay on Electrolyte Concentration and Electrolyte Species. As an example, Figure 2 shows the SH electric field $E_{2 \omega}$ as a function of time $t$ for the $\mathrm{NaCl}$ electrolyte solution at different concentrations. Figure 3 represents the temporal profiles of $E_{2 \omega}(t)$ for different kinds of electrolytes at the same electrolyte concentration. It is readily seen that the SH field decay behavior depends on the electrolyte species and its concentration.

$E_{2 \omega}(t)$ was fitted to a single-exponential function

$$
E_{2 \omega}(t)=a_{1}+a_{2} \exp (-t / \tau)
$$

where $\tau$ is the time constant of the decay of the $\mathrm{SH}$ electric field, and, $a_{1}$ and $a_{2}$ are proportionality constants. It can be inferred from eq 1 that the sum of $a_{1}$ and $a_{2}$ is proportional to the number of adsorbate molecules at the outer surface of the liposome bilayer at $t=0$, there being no adsorbate molecules at the inner surface at that time.

The results are summarized in Table 1 and Table 2. It should be mentioned that each fitting parameter represents an average of 7-10 experimental runs with the same liposome sample. In addition, the same experiment has been repeated at least twice by preparing a fresh liposome sample under the same experimental conditions. The repeated data are also included in the tables. From the tables it can be seen that the reproducibility of our experiments is fair, i.e., $\pm 10 \%$. The averaged time constants for SH field decay are plotted against electrolyte concentrations in Figure 4, from which we summarize the following results.

First, for the samples containing inorganic electrolytes (i.e., $\mathrm{NaCl}$ and $\mathrm{NaBr}$ ), the decay time of the $\mathrm{SH}$ field increases with increasing electrolyte concentration, i.e., the transport rate of MG becomes slower as the electrolyte concentration increases. Second, there is no dependence of the decay time for the $\mathrm{SH}$ field on the concentration of the organic electrolytes, (i.e., $\mathrm{NaCitrate}$ and $\mathrm{NaEtSO}_{3}$ ). The decay times for these two organic electrolytes are the same within experimental error. The third interesting result is that the decay time of the $\mathrm{SH}$ field depends on the electrolyte species at the same electrolyte concentration. It is the largest with $\mathrm{NaCl}$, smaller with $\mathrm{NaBr}$, and smallest with the two organic electrolytes. Last, if the electrolyte concentration is low enough $(1 \mathrm{mM})$, the decay time of the $\mathrm{SH}$ field converges to the same value for the four electrolytes, i.e., $\mathrm{MG}$ crosses the bilayer with almost the same rate at $1 \mathrm{mM}$ for the four electrolytes.

In this study, we changed electrolytes by varying only the anions (i.e., $\mathrm{Cl}^{-}, \mathrm{Br}^{-}$, Citrate ${ }^{-}$, and $\mathrm{EtSO}_{3}{ }^{-}$) and keeping the same cation (i.e., $\mathrm{Na}^{+}$). The above observations are therefore ascribed to the effects of the different anions, in what we call the counterion effect.

C. Electrolyte Concentration Dependence of the Initial MG Adsorption on Liposome Outer Layer. The sum of the 

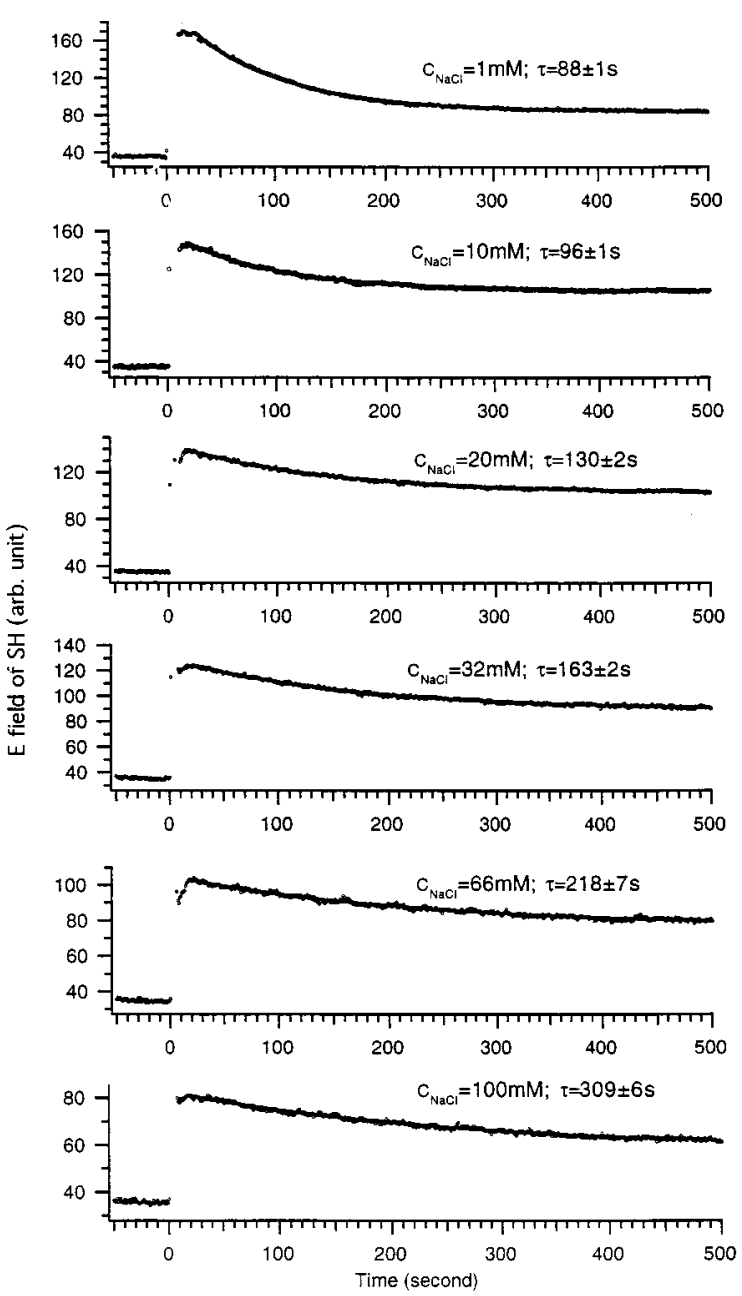

Figure 2. Measured and fitted time evolution (solid line) of SH electric field upon injection of liposomes into a MG solution at different concentrations of $\mathrm{NaCl}$ at $\mathrm{pH} 4.0$

two fitting parameters $\left(a_{1}+a_{2}\right)$ is proportional to the number of adsorbates at the outer surface of the liposome bilayer at $t=$ $0, N_{\mathrm{o}}(t=0)$. The correlation of $\left(a_{1}+a_{2}\right)$ with electrolyte concentration is plotted in Figure 5. It is shown that for the four electrolytes studied here, the change of $N_{\mathrm{o}}(t=0)$ with electrolyte concentration follows a similar trend. More specifically, the population density of MG adsorbed at $t=0$ at the outer surface of the liposome bilayer decreases with electrolyte concentration.

The surface of DOPG liposome is negatively charged. The negative surface potential acts as a driving force to bring the positively charged MG adsorbates from the bulk solution to the outer surface of bilayer. The increase of electrolyte concentration leads to a decrease in the magnitude of the surface potential due to increased screening of the charged liposome surface by the electrolyte. ${ }^{29}$ Thus, a lowering of the number of MG cations adsorbed at the outer surface can be expected. This result is consistent with the result we obtained previously, ${ }^{31}$ i.e., the dependence of the MG adsorption on the liposome surface charge density. It is also found that at the same electrolyte concentration the values of $N_{\mathrm{o}}(t=0)$ are close to each other for the four electrolytes. This indicates that the surface potential is about the same for the four cases. It can be noted from these results that the different counterions affect the initial adsorption of MG in the same way.

D. Possible Explanation of the Counterion Effect on MG Transport. It is obvious that the observed counterion species
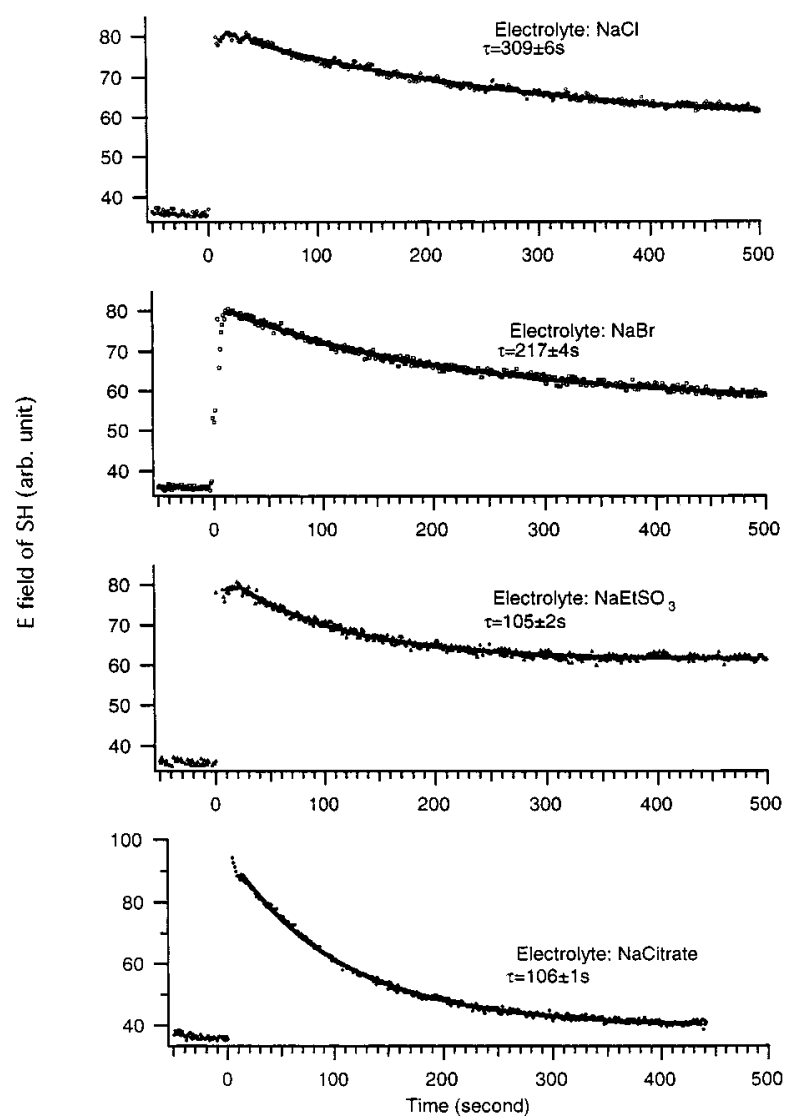

Figure 3. Measured and fitted time evolution (solid line) of SH electric field upon injection of liposomes into a pH 4.0 MG solution containing different electrolytes at the fixed concentration of $100 \mathrm{mM}$.

dependence of MG transport across the liposome bilayer cannot be explained by a macroscopic continuum mechanism. Instead a molecular picture about the interaction between the transport ion $\mathrm{MG}$ and the counterion (i.e., $\mathrm{Cl}^{-}, \mathrm{Br}^{-}, \mathrm{Citrate}^{-}$, or $\mathrm{EtSO}_{3}{ }^{-}$) must be involved. In the following we will provide a mechanism to understand our experimental results.

A possible model is that the counterion stabilizes the $\mathrm{MG}$ molecules outside of the bilayer, perhaps by forming loose ion pairs with MG in the bulk aquous phase through electrostatic interactions. Such a stabilization of MG can lower the free energy of MG in the outer region with respect to the higher free energy in the central hydrophobic region of the bilayer. Thus, the free energy of activation for MG to enter the bilayer is increased, which therefore affects the transport rate of MG across the liposome bilayer. The effects of the electrolyte on the electrical free energy of an ion (i.e., MG in this study) can be described by the Debye-Hückel theory, in which the ion is treated as a conducting sphere surrounded by an electrolyte (anions in this study) atmosphere. On the basis of this theory, the total electrical free energy of a spherical ion of radius $b$ is given as

$$
F=\frac{z^{2} e^{2}}{2 D}\left(\frac{1}{b}-\frac{\kappa}{1+\kappa a}\right)
$$

where $z$ is the valence of the ion under consideration, $e$ is the electron charge, $D$ is dielectric constant for the solution, and $\kappa$ is defined as

$$
\kappa=\sqrt{\frac{4 \pi e^{2}}{D k T} \cdot \frac{2 N}{1000} \cdot I}
$$


12820 J. Phys. Chem. B, Vol. 105, No. 51, 2001

Shang et al.

TABLE 1: Time Constants of the SH Field Decay and Their Averages with Four Electrolytes at Different Concentrations

\begin{tabular}{|c|c|c|c|c|c|c|c|c|}
\hline \multirow{2}{*}{$\begin{array}{c}\text { electrolyte } \\
\text { concentration }(\mathrm{mM})\end{array}$} & \multicolumn{2}{|c|}{$\tau_{\mathrm{NaCl}}(\mathrm{s})$} & \multicolumn{2}{|c|}{$\tau_{\mathrm{NaBr}}(\mathrm{s})$} & \multicolumn{2}{|c|}{$\tau_{\text {NaCitrate }}(\mathrm{s})$} & \multicolumn{2}{|c|}{$\tau_{\mathrm{NaEtSO}_{3}}(s)$} \\
\hline & experiment & average & experiment & $\overline{\text { average }}$ & experiment & average & experiment & average \\
\hline 1 & $\begin{array}{l}88 \pm 5 \\
91 \pm 7\end{array}$ & $89 \pm 4$ & $\begin{array}{l}94 \pm 15 \\
87 \pm 10\end{array}$ & $90 \pm 9$ & $\begin{array}{l}97 \pm 6 \\
97 \pm 4\end{array}$ & $97 \pm 4$ & $\begin{array}{l}86 \pm 8 \\
96 \pm 11\end{array}$ & $91 \pm 7$ \\
\hline 10 & $\begin{aligned} 119 & \pm 8 \\
96 & \pm 16\end{aligned}$ & $107 \pm 11$ & $\begin{array}{l}91 \pm 9 \\
95 \pm 8\end{array}$ & $93 \pm 6$ & $\begin{array}{r}122 \pm 8 \\
92 \pm 7\end{array}$ & $107 \pm 5$ & $\begin{array}{r}114 \pm 19 \\
102 \pm 10 \\
96 \pm 10\end{array}$ & $104 \pm 8$ \\
\hline 20 & $\begin{array}{l}132 \pm 15 \\
111 \pm 12\end{array}$ & $121 \pm 10$ & $\begin{array}{r}96 \pm 10 \\
114 \pm 15\end{array}$ & $105 \pm 9$ & $\begin{array}{l}89 \pm 5 \\
87 \pm 7\end{array}$ & $88 \pm 4$ & $\begin{array}{r}108 \pm 14 \\
98 \pm 14\end{array}$ & $103 \pm 10$ \\
\hline 32 & $\begin{array}{l}190 \pm 19 \\
160 \pm 26\end{array}$ & $175 \pm 16$ & $\begin{array}{l}127 \pm 9 \\
114 \pm 27 \\
117 \pm 15\end{array}$ & $119 \pm 11$ & $\begin{array}{l}99 \pm 14 \\
86 \pm 7\end{array}$ & $92 \pm 8$ & $\begin{array}{r}134 \pm 17 \\
106 \pm 19 \\
92 \pm 20\end{array}$ & $111 \pm 11$ \\
\hline 66 & $\begin{array}{l}241 \pm 41 \\
226 \pm 23 \\
206 \pm 11 \\
202 \pm 19 \\
214 \pm 11\end{array}$ & $218 \pm 13$ & $\begin{array}{l}152 \pm 25 \\
177 \pm 24\end{array}$ & $165 \pm 17$ & $\begin{array}{l}118 \pm 5 \\
108 \pm 15\end{array}$ & $113 \pm 8$ & $\begin{array}{l}132 \pm 15 \\
105 \pm 21\end{array}$ & $118 \pm 13$ \\
\hline 100 & $\begin{array}{l}363 \pm 41 \\
328 \pm 22 \\
289 \pm 31\end{array}$ & $327 \pm 19$ & $\begin{array}{l}216 \pm 34 \\
217 \pm 35\end{array}$ & $216 \pm 24$ & $\begin{array}{l}114 \pm 21 \\
105 \pm 13\end{array}$ & $109 \pm 12$ & $\begin{array}{l}112 \pm 18 \\
102 \pm 10 \\
110 \pm 12\end{array}$ & $108 \pm 8$ \\
\hline
\end{tabular}

TABLE 2: Sums of the Fitted Parameters $\left(a_{1}+a_{2}\right)$ and Their Averages with Four Electrolytes at Different Concentrations

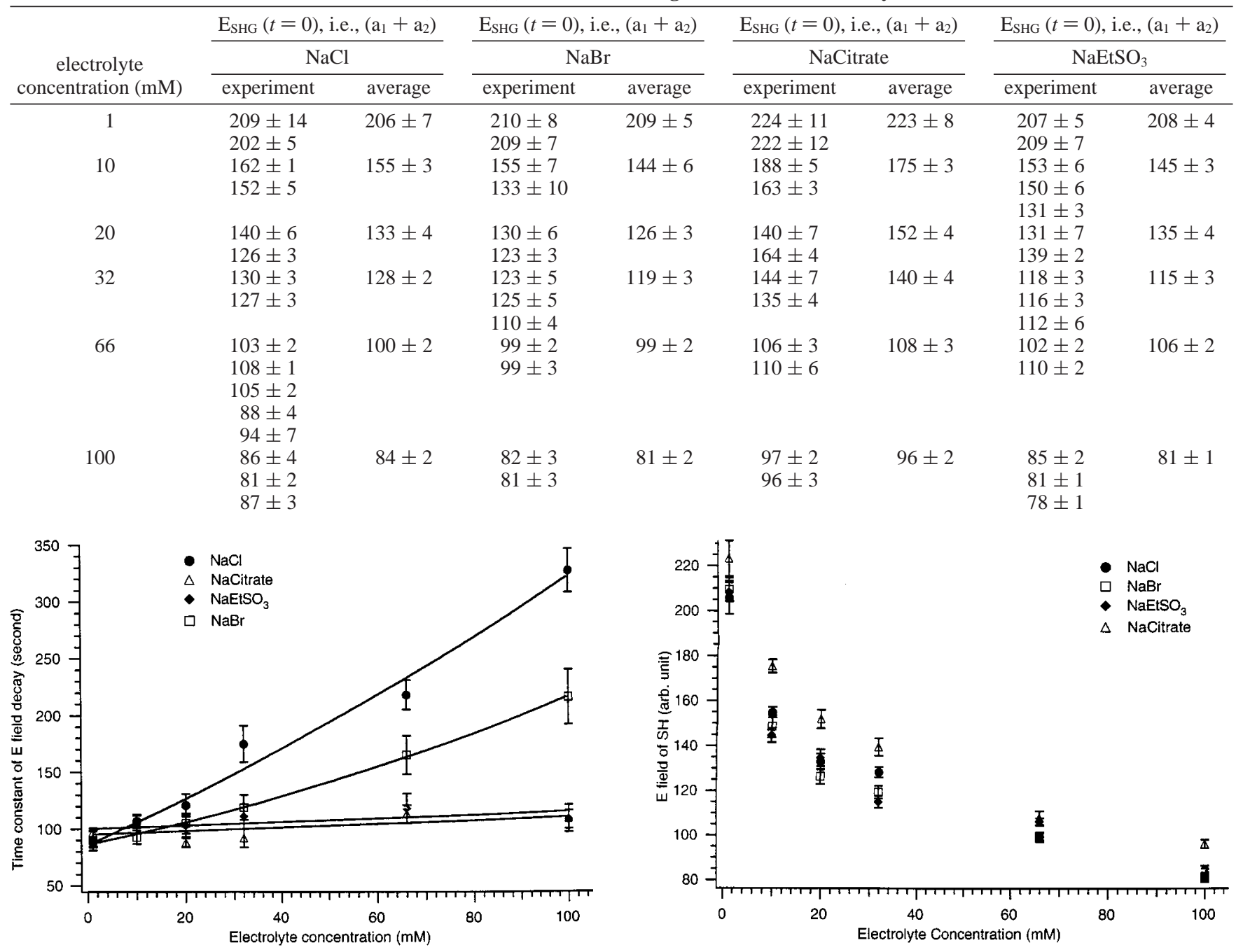

Figure 4. Averaged time constant of the decay of the SH electric field versus electrolyte concentration for different electrolytes at $\mathrm{pH} 4.0$. The solid lines are used for guidance.

with $k$ Boltzmann constant, $T$ temperature, $N$ Avogadro's number, and $I$ ionic strength. The variable $a$ represents the distance of closest approach between the center of the ion under consideration and the electrolyte ion. For the inorganic anions in this study, It is likely to be smaller than the sum of the radii

Figure 5. Averaged sum of two fitted parameters, $a_{1}+a_{2}$ (i.e., $\mathrm{SH}$ electrolyte field at $t=0$ ) versus electrolyte concentration for different electrolytes at $\mathrm{pH} 4.0$.

of the MG and the inorganic anion because the $\mathrm{Cl}^{-}$and $\mathrm{Br}^{-}$ can be located in a pocket of the central charged carbon of $\mathrm{MG}$ molecule. For the organic anions in this study, the steric hindrance by the aromatic groups interferes with organic anion being located in the central carbon pocket. Therefore, $a$ cannot 


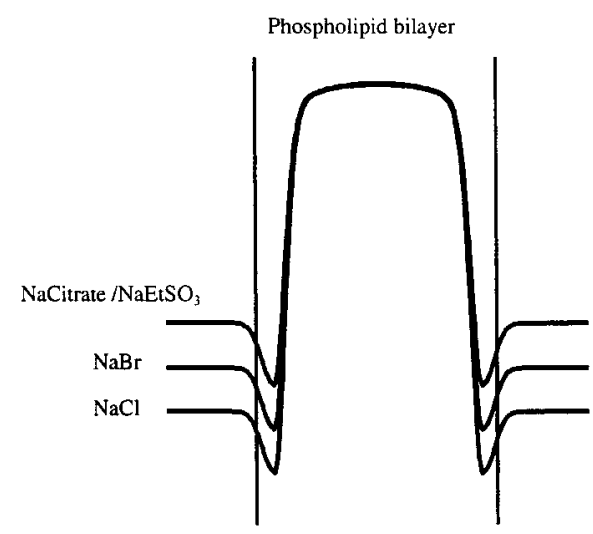

Figure 6. Schematic energy profiles of MG of the four electrolytes at the same electrolyte concentration.

be smaller than the sum of the radii of the MG and the organic anion. The first term in the parenthesis of eq 4 represents the free energy of a spherical ion immersed in the medium of dielectric constant $D$ and only depends on the property of ion itself (i.e., ion size). The second term in the parenthesis reflects the electrostatic interaction between the ion and its surrounding ions and is related to both the center-center distance between the ion and its closest neighboring ion (i.e., $a$ ) and the ionic strength of the electrolyte (i.e, $I$ ). Because the second term is negative, the electrostatic interaction between the ion and its surrounding ions tends to lower the free energy of the ion.

For the system we studied, the minimum distance between the MG molecule and the anion (counterion) depends on the anion size and steric structure of MG. As shown in Scheme 1, the MG molecule has a positively charged central carbon atom that is buried inside of three aromatic groups, arranged in a propeller-like geometry to minimize repulsive interactions between the hydrogen atoms on the neighboring aromatic rings. In the four salts used in this study, the sizes of their anions varies in the order of $\mathrm{Cl}^{-}<\mathrm{Br}^{-}<\mathrm{Citrate}^{-}$and $\mathrm{EtSO}_{3}{ }^{-} . \mathrm{Cl}^{-}$ can get closest to the central carbon of $\mathrm{MG}$, possibly forming a loose ion pair, and $\mathrm{Br}^{-}$the next, while Citrate ${ }^{-}$and $\mathrm{EtSO}_{3}{ }^{-}$ cannot approach as close to the central charged carbon of MG due to their bigger ion sizes. Therefore the distance of closest approach between $\mathrm{MG}$ and anion varies in the same order as

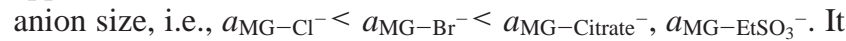
can be implied from eq 4 that the free energy of MG in the presence of the inorganic anion is lower than that in the presence of the larger organic anions. For the two inorganic anions, the free energy of $\mathrm{MG}$ with $\mathrm{Cl}^{-}$is lower than that with $\mathrm{Br}^{-}$. As a result, the energy barrier that $\mathrm{MG}$ has to overcome in going from the outside aqueous electrolyte region to the inner hydrocarbon region is the highest with $\mathrm{Cl}^{-}$, lower with $\mathrm{Br}^{-}$, and the lowest with the organic anions. The time constant for MG transport would be longest for $\mathrm{Cl}^{-}$, next longest for $\mathrm{Br}^{-}$, and shortest for the organic anions. The energy barrier for moving MG from the aqueous bulk electrolyte solution to the inside of the hydrophobic bilayer using a Born energy model is described in detail in ref 40. Figure 6 schematically illustrates how different anion species affect the energy barrier for MG in going from an aqueous electrolyte bulk solution to the interior of the bilayer.

The concentration dependence of the MG transport rate in the two inorganic electrolytes can also be explained with this mechanism. The increase of the electrolyte concentration leads to an increase of the number of anions $\left(\mathrm{Cl}^{-}\right.$or $\left.\mathrm{Br}^{-}\right)$which are close to the central carbon of MG molecules, and increases the number of loose ion pairs. As a result, $\mathrm{MG}$ would be more stabilized and thus have a lower free energy. Such an effect can also be understood in terms of the Debye-Hückel model. As shown in eq 4, the increase of electrolyte concentration (i.e., $\kappa)$ leads to the increase of the electrical energy accounting for the interaction between MG and its surrounding anions (i.e., the second term in the parenthesis of eq 4), thereby lowers the free energy of MG. It is reasonable to assume that the potential inside the bilayer is independent of electrolyte concentration. One might then expect that the energy barrier for MG transporting across the bilayer becomes higher with increasing electrolyte concentration. Thus MG crosses the bilayer at a slower rate when the electrolyte concentration is higher (see Figure 2).

The weaker dependence of the organic anion time constants on electrolyte concentration suggests that there is no significant stabilization of MG perhaps because their distances of closest approach to the central carbon of the MG molecule are too large to form loose ion pairs with MG. It would then follow that the organic anion time constants should be close to each other, which is consistent with our finding.

In this work, we also found that at low electrolyte concentration $(1 \mathrm{mM})$, the transport rate of $\mathrm{MG}$ approaches the same value for all of the anions. This can be understood as follows. At an electrolyte concentration of $1 \mathrm{mM}$, the electrostatic interaction between $\mathrm{MG}$ and the anions can be neglected for both organic and inorganic anions because $1 / \kappa(=96 \AA)$ is much larger than the ion radius of MG. In this case, the electrical free energies of MG in the presence of the four anionic species are equal. Thus, it would be expected that the transport rates of MG in the presence of the four electrolytes might approach the same value at low electrolyte concentrations, which is consistent with our experimental results.

\section{Conclusions}

In this study, we have observed for the first time, to our knowledge, the effect of the counterion, which is an anion, on the molecular transport of MG, an organic cation, across the bilayer of a unilamellar dioleoylphosphatidylglycerol (DOPG) liposome $(\sim 105 \mathrm{~nm})$. The following four results are obtained from the second harmonic experiments: (1) With the two inorganic anions, $\mathrm{Cl}^{-}$and $\mathrm{Br}^{-}$, the time constant for $\mathrm{MG}$ transport increases with the concentration of counterions (i.e., $\mathrm{Cl}^{-}$or $\mathrm{Br}^{-}$). (2) There is no observable dependence of the transport time constants of $\mathrm{MG}$ on the concentration of the counterion with the two organic counterions, Citrate ${ }^{-}$and $\mathrm{EtSO}_{3}{ }^{-}$. (3) At the same counterion concentration, the transport rate of MG across the liposome bilayer is the slowest with $\mathrm{Cl}^{-}$, faster with $\mathrm{Br}^{-}$, and the fastest with $\mathrm{Citrate}^{-}$and $\mathrm{EtSO}_{3}{ }^{-}$. The transport rate for the $\mathrm{Cl}^{-}$at $100 \mathrm{mM}$ is three times slower than that for the organic counterions. (4) When the counterion concentration is low enough $(1 \mathrm{mM})$, the transport rate of $\mathrm{MG}$ across the membrane converges to the same value for the four electrolytes used in this study.

A tentative mechanism is one in which the MG free energy outside the liposome is lowered by the formation of loose ion pairs. It is highest with $\mathrm{Cl}^{-}$because of its small size, with $\mathrm{Br}^{-}$ next, and then the organic anions not forming ion pairs at all. The activation energies for crossing the barrier are in the order $\mathrm{Cl}^{-}>\mathrm{Br}^{-}>$Citrate ${ }^{-}$and $\mathrm{EtSO}_{3}{ }^{-}$. Thus the transport time would be longest for $\mathrm{Cl}^{-}$, next $\mathrm{Br}^{-}$, and then the organic anions. The concentration dependence of the MG transport rate with the two inorganic anions can also be understood with this mechanism. The increase of the inorganic electrolyte concentration tends to increase the number of loose ion pairs, therefore further lowering the free energy of MG outside the liposome. 
Acknowledgment. The authors wish to thank the National Science Foundation and the Division of Chemical Science, Office of Basic Energy Sciences of the Department of Energy for their support. The authors also wish to thank Profs. B. Honig and B. Roux for valuable discussions.

\section{References and Notes}

(1) Gennis, R. B. Biomembrane: Molecular Structure and Function; Springer-Verlag: New York, 1989.

(2) Lasic, D. D. Limposomes from Physics to Applications; Elsevier: Amsterdam, 1993.

(3) Limposomes as Tools in Basic Research and Industry; Philiport, J. R., Schuber, F., Eds.; CRC Press: 1995.

(4) Paregian, V. A. Nature 1969, 221, 844.

(5) MacDonald, R. C. Biochim. Biophys. Acta 1976, 448, 193.

(6) Deamer, D. W.; Bramhall, J. Chem. Phys. Lipids 1986, 40, 167

(7) Hamilton, R. T.; Kaler, E. W. J. Phys. Chem. 1990, 94, 2560.

(8) Paula, S.; Volkov, A. G.; Van Hoek, A. N.; Haines, T. H.; Deamer, D. W. Biophys. J. 1996, 70, 339 .

(9) Kaiser, S.; Hoffmann, H. J. Colloid Interface Sci. 1996, 184, 1.

(10) Paula, S.; Volkov, A. G.; Deamer, D. W. Biophys. J. 1998, 74 319.

(11) Bordi, F.; Cametti, C.; Naglieri, A. Biophys. J. 1998, 74, 1358 231.

(12) Bordi, F.; Cametti, C.; Naglieri, A. Colloids Surf., A 1999, 159, 225

(14) Vilson, M. A.; Pohorille, A. J. Am. Chem. Soc. 1996, 118, 6580.

(15) Xiang, T. X.; Bradley, D. A. Biophys. J 1992, 72, 223. 64.

(16) Xiang, T. X.; Bradley, D. A. Biochim. Biophys. Acta 1998, 1370,
(17) Cafiso, D. S.; Hubbell, W. L. Biophys. J. 1983, 44, 49.

(18) Chakrabarti, A. C.; Clark-Lewis, I.; Cullis, P. R. Biochem. 1994, 33,8479 .

(19) Services, R. F. Sience 1994, 265, 316

(20) Shen, Y. R. Аnпи. Rev. Phys. Chem. 1989, 40, 327.

(21) Corn, R. M.; Higgins, D. A. Chem. Rev. 1994, 94, 107.

(22) Eisenthal, K. B. Chem. Rev. 1996, 96, 1343.

(23) Eisenthal, K. B. J. Phys. Chem. 1996, 100, 12997.

(24) Miranda, P. B.; Shen, Y. R. J. Phys. Chem. 1999, 103, 3292.

(25) Shen, Y. R. Appl. Phys. B 1999, 68, 295.

(26) Wang, H.; Yan, E. C. Y.; Borguet, E.; Eisenthal, K. B. Chem. Phys. Lett. 1996, 259, 15.

(27) Wang, H.; Yan, E. C. Y.; Liu, Y.; Eisenthal, K. B. J. Phys. Chem. $B$ 1998, 102, 4446.

(28) Srivastava, A.; Eisenthal, K. B. Chem. Phys. Lett. 1998, 292, 345.

(29) Yan, E. C. Y.; Liu, Y.; Eisenthal, K. B. J. Phys. Chem. B 1998, 102,6331 .

(30) Liu, Y.; Dadap, J. I.; Zimdars, D.; Eisenthal, K. B. J. Phys. Chem. B 1999, 103, 2480 .

(31) Liu, Y.; Yan, E. C. Y.; Eisenthal, K. B. Submitted to Biophys. J.

(32) Yan, E. C. Y.; Eisenthal, K. B. J. Phys. Chem. B 1999, 103, 6056.

(33) Yan, E. C. Y.; Eisenthal, K. B. Biophys. J. 2000, 79, 898.

(34) Buster, D. C.; Hinton, J. F.; Millett, F. S.; Shungu, B. C. Biophys. J. 1988, 50, 145 .

(35) Cafiso, D. S. Methods Enzymol. 1989, 172, 331.

(36) Eidelman, O.; Cabantchik, Z. I. Biochim. Biophys. Acta 1989, 988 , 310 .

(37) Mayer, L. D.; Hope, M. J.; Cullis, P. R. Biochim, Biophys. Acta 1986, 858,161

(38) Kerker, M. The Scattering of Light and other Electromagnetic Radiation, 2nd Ed.; Academic: New York: 1970.

(39) Engelbert, H. P.; Lawaczeck, R. Chem. Phys. Lipids 1985, 38, 365.

(40) Honig, B. H. Аnпи. Rev. Biophys. Chem. 1986, 15, 163. 